\title{
DEFORMATIONS OF DUPIN HYPERSURFACES
}

\author{
ULRICH PINKALL AND GUDLAUGUR THORBERGSSON
}

(Communicated by Jonathan M. Rosenberg)

Dedicated to Wilhelm Klingenberg on his 65 th birthday

\begin{abstract}
Examples are given of compact proper-Dupin hypersurfaces that are not Lie equivalent to an isoparametric hypersurface.
\end{abstract}

\section{DUPIN HYPERSURFACES}

A question raised by many authors is whether a compact proper-Dupin hypersurface is equivalent to an isoparametric hypersurface under a Lie transformation. Here we say that a hypersurface or more generally a submanifold of $S^{n+1}$ is proper-Dupin if the principal curvatures have globally constant multiplicities and are constant along the corresponding curvature leaves which lie in the unit normal bundle if the codimension is greater than one. One can prove that a submanifold is proper-Dupin if and only if a small tube around it is a proper-Dupin hypersurface. Notice that the terminology has not yet been standardized. Thus our proper-Dupin hypersurfaces are just called Dupin in [CR], [GH], [Mi 1,2], [MO] and [Th]. Our terminology is essentially that of [Pi]. For the concept of Lie transformations and Lie sphere geometry in general we refer to $[\mathrm{CC}]$ and $[\mathrm{Pi}]$.

An evidence for a positive answer to the question is that the geometry and topology of proper-Dupin hypersurfaces and isoparametric hypersurfaces are very similar. E.g., for both classes the number $g$ of principal curvatures can only be $1,2,3,4$ or 6 and the dimension of the $\mathbf{Z}_{2}$-cohomology ring is $2 g$, see [Mü] and [Th]. See also [GH] for further similarities in the topology of these two classes of hypersurfaces.

The question has been answered positively for the cases $g=1$ (trivial), $g=2[\mathrm{CR}]$, see also [Pi], and $g=3[\mathrm{Mi} 1]$. It will be shown in the present paper that the answer is negative for $g=4$. More precisely, we will find a oneparameter deformation $M(t)$ of any isoparametric hypersurface $M$ of Clifford

Received by the editors October 10, 1988 and, in revised form, December 19, 1988.

1980 Mathematics Subject Classification (1985 Revision). Primary 53C42, 53A40.

Research begun during a visit of the second author at the Technische Universität Berlin, supported by the exchange program of GMD, the Federal Republic of Germany, and CNPq, Brazil. 
type [FKM] such that all hypersurfaces in the family are proper-Dupin, but no one except $M$ itself is Lie equivalent to $M$.

We now describe the one-parameter deformation in case of the isoparametric hypersurfaces of Clifford type based on the real numbers, i.e., on the Clifford algebra $\mathscr{C}_{0}=\mathbf{R}$. These isoparametric hypersurfaces are tubes around the Stiefel manifolds $V_{2, k}(\mathbf{R}) \subset S^{2 k-1} \subset \mathbf{R}^{k} \oplus \mathbf{R}^{k}$ of 2-frames of orthogonal vectors of length $1 / \sqrt{2}$. Notice that the affine transformation $T_{\alpha, \beta}: \mathbf{R}^{k} \oplus \mathbf{R}^{k} \rightarrow \mathbf{R}^{k} \oplus \mathbf{R}^{k}$; $(u, v) \rightarrow \sqrt{2}(\alpha u, \beta v), \alpha^{2}+\beta^{2}=1$, maps $V_{2, k}\left(\mathbf{R}^{k}\right)$ into another submanifold $V_{\alpha, \beta}:=T_{\alpha, \beta}\left(V_{2, k}\left(\mathbf{R}^{k}\right)\right)$ of $S^{2 k-1}$. We then show that the image $V_{\alpha, \beta}$ is properDupin. Small tubes around $V_{\alpha, \beta}$ are then also proper-Dupin. Now we consider the Lie curvature of these tubes. The Lie curvature is a function on a properDupin hypersurface with four different principal curvatures that is invariant under Lie transformations. For isoparametric hypersurfaces the Lie curvature is identically $1 / 2$. We then show for the tubes around $V_{\alpha, \beta}$ that the Lie curvature at a certain point is $\alpha^{2}$. As a consequence, these tubes can only be equivalent to an isoparametric hypersurface under a Lie transformation for $\alpha^{2}=1 / 2$.

In $\S 2$ we explain what we need about isoparametric hypersurfaces of Clifford type. In $\S 3$ we define the deformation and calculate the Lie curvature.

After this paper was finished, we received a preprint [MO] from Miyaoka and Ozawa with more examples of proper-Dupin hypersurfaces that are not Lie equivalent to isoparametric hypersurfaces. Their method also works for $g=6$, but only gives examples of Dupin hypersurfaces in $S^{7}$.

\section{Clifford 2-Frames}

There are three infinite series of homogeneous isoparametric hypersurfaces with $g=4$ principal curvatures in $S^{2 l-1}$. Most conveniently, these can be described as tubes around the real, complex or quaternionic Stiefel manifolds $V_{2, k}(\mathbf{R}), V_{2, k}(\mathbf{C}), V_{2, k}(\mathbf{H})$ of orthogonal 2-frames in $\mathbf{R}^{l}=\mathbf{R}^{k}, \mathbf{C}^{k}$ or $\mathbf{H}^{k}$. Here 'orthogonal' refers to the standard real, complex or quaternionic Hermitian scalar product $\langle,\rangle_{\mathbf{F}}$ in $\mathbf{R}^{k}, \mathbf{C}^{k}$ or $\mathbf{H}^{k}$. Here we assume $k \geq 3$ in the real case and $k \geq 2$ otherwise.

Let us look for example more closely at the notion of quaternionic orthogonality in $\mathbf{H}^{k}$. Let $E_{1}, E_{2}, E_{3}: \mathbf{H}^{k} \rightarrow \mathbf{H}^{k}$ denote left multiplication by $i, j$, $k$ respectively. Then a vector $v \in \mathbf{H}^{k}$ is quaternionically orthogonal to a vector $u \in \mathbf{H}^{k}$ if $v$ is real orthogonal to the one-dimensional quaternionic subspace of $\mathbf{H}^{k}$ spanned by $u$, i.e.,

$$
\langle u, v\rangle=\left\langle E_{1} u, v\right\rangle=\left\langle E_{2} u, v\right\rangle=\left\langle E_{3} u, v\right\rangle=0 .
$$

Here $\langle\rangle:, \mathbf{H}^{k} \times \mathbf{H}^{k} \rightarrow \mathbf{R}$ denotes the canonical real scalar product in $\mathbf{H}^{k}$, i.e., for all $w \in \mathbf{H}^{k}$ we have $\langle w, w\rangle_{\mathbf{H}}=\langle w, w\rangle$. Since $E_{1}, E_{2}, E_{3}$ are skew symmetric with respect to $\langle$,$\rangle , quaternionic orthogonality is a symmetric$ relation. 
The pairs $(u, v)$ of quaternionically orthogonal vectors in $\mathbf{H}^{k}$, such that both $u$ and $v$ have length $1 / \sqrt{2}$, form a submanifold $V_{2, k}(\mathbf{H})$ in $\mathbf{H}^{k} \oplus \mathbf{H}^{k}$ of codimension 6. In fact, $V_{2, k}(\mathbf{H})$ is contained in the unit sphere $S^{8 k-1}$. Taking a tube around $V_{2, k}(\mathbf{H})$ in $S^{8 k-1}$ we obtain a homogeneous isoparametric hypersurface. In fact, consider $\mathbf{H}^{k} \oplus \mathbf{H}^{k}$ as the space of quaternionic $(k, 2)$ matrices; $\mathrm{Sp}(2)$ acts on $M_{k, 2}(\mathbf{H})$ by multiplication from the right and $\mathrm{Sp}(k)$ acts on $M_{k, 2}(\mathbf{H})$ by multiplication from the left. Then

(i) $V_{2, k}(\mathbf{H})$ is an orbit of the $\mathrm{Sp}(2) \times \mathrm{Sp}(k)$-action described above.

(ii) The principal orbits of this action have codimension two in $\mathbf{H}^{k} \oplus \mathbf{H}^{k}$ [HL].

Hence all tubes around $V_{2, k}(\mathbf{H})$ are homogeneous isoparametric hypersurfaces in $S^{8 k-1}$.

The Clifford examples of isoparametric hypersurfaces found by Ferus, Karcher and Münzner [FKM] arise from a natural generalization of the above construction. Instead of the $E_{1}, E_{2}, E_{3}$ in the quaternionic case, consider any set $\left\{E_{1}, \ldots, E_{m-1}\right\}$ of orthogonal operators on $\mathbf{R}^{l}$ which satisfy

$$
E_{i} E_{j}+E_{j} E_{i}=-2 \delta_{i j} \text { Id } .
$$

Such sets $\left\{E_{1}, \ldots, E_{m-1}\right\}$ are in 1-1 correspondence with orthogonal representations of the Clifford algebra $\mathscr{C}_{m-1}$ of $\mathbf{R}^{m-1}$ endowed with a negative definite metric $[\mathrm{Hu}]$. Thus we call $\left\{E_{1}, \ldots, E_{m-1}\right\}$ with the mentioned properties a Clifford set.

Given a Clifford set $\mathscr{C}=\left\{E_{1}, \ldots, E_{m-1}\right\}$ acting on $\mathbf{R}^{l}$ we define two vectors $u, v \in \mathbf{R}^{l}$ to be Clifford orthogonal if

$$
\langle u, v\rangle=\left\langle E_{1} u, v\right\rangle=\cdots=\left\langle E_{m-1} u, v\right\rangle=0 .
$$

As above Clifford orthogonality is a symmetric relation. The pairs $(u, v)$ of Clifford orthogonal vectors $u, v$ satisfying

$$
\langle u, u\rangle=\langle v, v\rangle=1 / 2 \text {, }
$$

form a submanifold $V_{2}(\mathscr{C})$ in $S^{l}(1 / \sqrt{2}) \times S^{l}(1 / \sqrt{2}) \subset S^{2 l-1}$. We call $V_{2}(\mathscr{C})$ the Clifford-Stiefel manifold of $\mathscr{C}$-orthonormal 2 -frames in $\mathbf{R}^{l}$. Note that in contrast to the quaternionic case, the two numbers $m$ and $l$ are in general not sufficient to characterize the geometric properties of $V_{2}(\mathscr{C})$. One has to resort to the classification of orthogonal representations of Clifford algebras, see $[\mathrm{FKM}]$.

The following observation is essentially Lemma 3 in Wang [Wa]: The isoparametric hypersurfaces of Clifford type in $S^{2 l-1}$, see $[\mathrm{FKM}]$, are exactly the tubes in $S^{2 l-1}$ around $V_{2}(\mathscr{C})$, where $\mathscr{C}=\left\{E_{1}, \ldots, E_{m-1}\right\}$ ranges over all Clifford sets acting on $\mathbf{R}^{l}$ and satisfying $l \geq m+2$. To see this, notice that the polynomial $F: \mathbf{R}^{2 l} \rightarrow \mathbf{R}$ in [FKM, Satz 4.1] restricted to $S^{2 l-1}$ takes its maximum 
value 1 on the focal variety $M_{+}$, which consists of all $x \in S^{2 l-1}$ satisfying

$$
\left\langle P_{0} x, x\right\rangle=\cdots=\left\langle P_{m} x, x\right\rangle=0 \text {. }
$$

If we rewrite the $P_{i}$ in terms of the $E_{j}$ (see [FKM, 3.3]) and substitute $(u, v)$ for $x$ we see that these equations are equivalent to

$$
\begin{array}{r}
\langle u, u\rangle-\langle v, v\rangle=\langle u, v\rangle=0, \\
\left\langle E_{1} u, v\right\rangle=\cdots=\left\langle E_{m-1} u, v\right\rangle=0 .
\end{array}
$$

Clearly, these equations are just the defining equations for $V_{2}(\mathscr{C})$. Since the isoparametric hypersurfaces are tubes around $M_{+}$the observation is proved.

Knowing the three infinite series of homogeneous isoparametric families (tubes around $\left.V_{2, k}(\mathbf{R}), V_{2, k}(\mathbf{C}), V_{2, k}(\mathbf{H})\right)$ the most obvious examples of inhomogeneous isoparametric hypersurfaces are the tubes around $V_{2, k}(O)$, the Stiefel manifold of Cayley 2-frames in $O^{k}$. Here $k \geq 2$ and the Clifford set $\left\{E_{1}, \ldots, E_{7}\right\}$ is given by left multiplication of vectors in $O^{k}$ by the seven imaginary units $e_{1}, \ldots, e_{7}$ of $O$. The inhomogeneity of these examples follows from the classification of homogeneous isoparametric hypersurfaces [HL], [TT].

It might seem natural to try to define 'Stiefel manifolds' $V_{k}(\mathscr{C}), k \geq 3$. Such an attempt would fail for the following reason: The dimension of the space of all $w \in \mathbf{R}^{l}$ such that $w$ is Clifford orthogonal to both $u$ and $v$, $(u, v) \in V_{2}(\mathscr{C})$, may depend on the choice of $(u, v)$.

\section{Deformations of Clifford-Stiefel Manifolds}

In [Mi 2] Miyaoka defines the Lie curvature $\Psi$ of a proper-Dupin hypersurface $M$ with $g=4$ principal curvatures as follows. Let $\lambda_{1}<\lambda_{2}<\lambda_{3}<\lambda_{4}$ be the principal curvatures. The cross ratio

$$
\Psi=\frac{\left(\lambda_{1}-\lambda_{2}\right)\left(\lambda_{4}-\lambda_{3}\right)}{\left(\lambda_{1}-\lambda_{3}\right)\left(\lambda_{4}-\lambda_{2}\right)}
$$

is then called the Lie curvature of $M$. It is shown in [Mi 2] that $\Psi$ is invariant under Lie transformations. It follows from [Mü] that $\Psi \equiv 1 / 2$ for an isoparametric hypersurface.

Now let $M$ be a proper-Dupin submanifold with codimension at least two and $g=3$ principal curvatures $\lambda_{1}<\lambda_{2}<\lambda_{3}$. Then we define the Möbius curvature of $M$ at a given normal vector of $M$ as

$$
\Phi=\frac{\left(\lambda_{1}-\lambda_{2}\right)}{\left(\lambda_{1}-\lambda_{3}\right)} .
$$

Again it follows from [Mi 2] that $\Phi$ is invariant under Möbius transformations. Notice that a proper-Dupin submanifold with codimension at least two and $g=3$ principal curvatures can be thought of as a hypersurface with $g=4$ principal curvatures and one principal curvature infinite. In this sense the Möbius curvature $\Phi$ is a special case of the Lie curvature $\Psi$. The focal submanifolds of 
isoparametric hypersurfaces with $g=4$ satisfy $\Phi \equiv 1 / 2$. More precisely, their principal curvatures are $-1,0$, and 1 . Notice furthermore that the Möbius curvature of a proper-Dupin submanifold $M$ of codimension at least two in the direction of a normal vector $\xi$ is equal to the Lie curvature of a tube around $M$ at the point where the great circle tangent to $\xi$ meets the tube.

Let now $\mathscr{C}=\left\{E_{1}, \ldots, E_{m-1}\right\}$ be a Clifford set acting on $\mathbf{R}^{l}, V_{2}(\mathscr{C}) \subset S^{2 l-1}$ the correpsonding Clifford-Stiefel manifold. Notice that $V_{2}(\mathscr{C})$ is contained in the set of all $(u, v) \in \mathbf{R}^{2 l}$ satisfying

$$
\begin{aligned}
\langle u, u\rangle=\langle v, v\rangle & =1 / 2, \\
\langle u, v\rangle & =0 .
\end{aligned}
$$

Thus given $\alpha, \beta \in \mathbf{R}_{+}$with $\alpha^{2}+\beta^{2}=1$ the image $V_{2}^{\alpha, \beta}(\mathscr{C})$ of $V_{2}(\mathscr{C})$ under the linear map

$$
T_{\alpha, \beta}: \mathbf{R}^{2 l} \rightarrow \mathbf{R}^{2 l} ; \quad(u, v) \rightarrow \sqrt{2}(\alpha u, \beta v)
$$

is again contained in $S^{2 l-1}$. Furthermore, $V_{2}^{\alpha, \beta}(\mathscr{C})$ is also proper-Dupin. To prove this, notice that a hypersphere in $S^{2 l-1}$ that touches $V_{2}(\mathscr{C})$ along a curvature surface $S$ lies in a hyperplane $H \in \mathbf{R}^{2 l}$ that is tangent to $V_{2}(\mathscr{C})$ along $S$. The image $T_{\alpha, \beta}(H)$ cuts $S^{2 l-1}$ in a hypersphere that touches $V_{2}^{\alpha, \beta}(\mathscr{C})$ along $T_{\alpha, \beta}(S)$. It follows that $T_{\alpha, \beta}(S)$ is a curvature leaf. We have thus proved that $V_{2}^{\alpha, \beta}(\mathscr{C})$ is proper-Dupin with $g=3$ principal curvatures.

We now show that there is a normal vector $\xi$ to $V_{2}^{\alpha, \beta}(\mathscr{C})$ such that the Möbius curvature of $V_{2}^{\alpha, \beta}(\mathscr{C})$ in the direction of $\xi$ is equal to $\alpha^{2}$. This will imply for $\alpha^{2} \neq 1 / 2$ that the tubes around $V_{2}^{\alpha, \beta}(\mathscr{C})$ are not Lie equivalent to isoparametric hypersurfaces.

Let $(u, v) \in V_{2}^{(\alpha, \beta}(\mathscr{C})$ be any point. We have

$$
V_{2}^{(\alpha, \beta}(\mathscr{C}) \subset f^{-1}(0) \cap g^{-1}(0)
$$

where $f, g: S^{2 l-1} \rightarrow \mathbf{R}$ are given by

$$
\begin{aligned}
& f(u, v)=\frac{\beta}{\alpha}\langle u, u\rangle-\frac{\alpha}{\beta}\langle v, v\rangle, \\
& g(u, v)=\langle u, v\rangle .
\end{aligned}
$$

Thus the gradients

$$
\begin{aligned}
\xi & =\left(\frac{\beta}{\alpha} u,-\frac{\alpha}{\beta} v\right), \\
\eta & =(v, u)
\end{aligned}
$$

of $f$ and $g$ provide two unit normal vectors to $V_{2}^{\alpha, \beta}(\mathscr{C})$ at $(u, v)$. Choose $x, y \in \mathbf{R}^{l}$ such that

$$
\left.\begin{array}{lll}
\|x\|=\alpha, & \left\langle x, E_{i} v\right\rangle=0, & \langle x, u\rangle=0 \\
\|y\|=\beta, & \left\langle y, E_{i} u\right\rangle=0, & \langle y, v\rangle=0
\end{array}\right\} \quad i=0, \ldots, m-1,
$$


where $E_{0}=\mathrm{Id}$, and define curves $\gamma_{x}, \gamma_{y}, \gamma_{z}$ in $V_{2}^{\alpha, \beta}(\mathscr{C})$ through $(u, v)$ as follows:

$$
\begin{aligned}
& \gamma_{x}(t)=(\cos t u+\sin t x, v), \\
& \gamma_{y}(t)=(u, \cos t v+\sin t y), \\
& \gamma_{z}(t)=\left(\cos t u+\frac{\alpha}{\beta} \sin t v,-\frac{\beta}{\alpha} \sin t u+\cos t v\right) .
\end{aligned}
$$

It is straightforward to check that these curves lie in $V_{2}^{\alpha, \beta}(\mathscr{C})$. Along $\gamma_{x}$ the normal vector field $\xi$ is given by

$$
\xi(t)=\left(\frac{\beta}{\alpha}(\cos t u+\sin t x),-\frac{\alpha}{\beta} v\right) .
$$

The tangent vector $\gamma_{x}^{\prime}(0)$ provides at $\gamma_{x}(0)=(u, v)$ a principal direction corresponding to the principal curvature

$$
\lambda_{x}=\frac{\beta}{\alpha}
$$

since

$$
\xi^{\prime}(0)=\left(\frac{\beta}{\alpha} x, 0\right)=\frac{\beta}{\alpha} \gamma_{x}^{\prime}(0) .
$$

Similarly, $\gamma_{y}^{\prime}(0)$ corresponds to the principal curvature

$$
\lambda_{y}=-\frac{\alpha}{\beta} \text {. }
$$

Finally, along $\gamma_{z}$ we have

$$
\begin{aligned}
\xi(t) & =\left(\frac{\beta}{\alpha}\left(\cos t u+\frac{\alpha}{\beta} \sin t v\right),-\frac{\alpha}{\beta}\left(-\frac{\beta}{\alpha} \sin t u+\cos t v\right)\right), \\
\xi^{\prime}(0) & =(v, u)=\eta,
\end{aligned}
$$

which means that $\gamma_{=}^{\prime}(0)$ lies in the kernel of the shape operator of $\xi$, so

$$
\lambda_{z}=0 \text {. }
$$

Having computed all three different principal curvatures in the direction of $\xi$, we find that the Möbius curvature $\Phi$ of $V_{2}^{(\alpha, \beta}(\mathscr{C})$ at $\xi$ is

$$
\Phi=\frac{\lambda_{y}-\lambda_{z}}{\lambda_{y}-\lambda_{x}} \alpha^{2} \text {. }
$$

We have thus proved that the tubes around $V_{2}^{(x, \beta}(\mathscr{C})$ are proper-Dupin hypersurfaces which are not Lie equivalent to any isoparametric hypersurface.

\section{REFERENCES}

[CC] T. E. Cecil and S.-S. Chern, Tautness and Lie sphere geometry, Math. Ann. 278 (1987), 381-399.

[CR] T. E. Cecil and P. J. Ryan, Tight and taut immersions of manifolds, Res. Notes Math. 107, Ptiman, Boston, London, Melbourne 1985. 
[FKM] D. Ferus, H. Karcher and H.-F. Münzner, Cliffordalgebren und neue isoparametrische Hyperflächen, Math. Z. 177 (1981), 479-502.

[GH] K. Grove and S. Halperin, Dupin hypersurfaces, group actions and the double mapping cylinder, J. Differential Geom. 26 (1987), 429-457.

[HL] W.-Y. Hsiang and H. B. Lawson, Minimal submanifolds of low cohomogeneity, J. Differential Geometry 5 (1971), 1-38.

[Hu] D. Husemoller, Fibre bundles, McGraw-Hill, New York, 1966.

[Mil] R. Miyaoka, Compact Dupin hypersurfaces with three principal curvatures, Math. Z. 187 (1984), 433-452.

[Mi2] _- Dupin hypersurfaces and a Lie invariant, (to appear in Kodai Math. J.).

[MO] R. Miyaoka and T. Ozawa, Construction of taut embeddings and Cecil-Ryan conjecture, preprint, Tokyo Institute of Technology and Nagoya University 1988.

[Mü] H.-F. Münzner, Isoparametrische Hyperflächen in Sphären I, Math. Ann. 251 (1980), 57-71; II, 256 (1981), 215-232.

[Pi] U. Pinkall, Dupin hypersurfaces, Math. Ann. 270 (1985), 427-440.

[TT] R. Takagi and T. Takahashi, On the principal curvatures of homogeneous hypersurfaces in a sphere, in Differential Geometry in Honor of K. Yano, Kinokuniya, Tokyo 1972, 469-481.

[Th] G. Thorbergsson, Dupin hypersurfaces, Bull. London Math. Soc. 15 (1983), 493-498.

[Wa] Q.-M. Wang, On the topology of Clifford isoparametric hypersurfaces, J. Differential Geometry 27 (1988), 55-66.

Fachbereich Mathematik, Technische Universität Berlin, Strasse des 17. Juni 135 , D-1000 BERLIN 12

Department of Mathematics, University of Notre Dame, Notre Dame, Indiana 46556 